This item was submitted to Loughborough's Research Repository by the author.

Items in Figshare are protected by copyright, with all rights reserved, unless otherwise indicated.

\title{
Depressive symptoms and obesity: instrumental variable analysis using mother-offspring pairs in the 1970 British Cohort Study
}

\section{PLEASE CITE THE PUBLISHED VERSION}

https://doi.org/10.1038/ijo.2016.143

\section{PUBLISHER}

(C) Nature Publishing Group

\section{VERSION}

AM (Accepted Manuscript)

\section{PUBLISHER STATEMENT}

This work is made available according to the conditions of the Creative Commons Attribution-NonCommercialNoDerivatives 4.0 International (CC BY-NC-ND 4.0) licence. Full details of this licence are available at: https://creativecommons.org/licenses/by-nc-nd/4.0/

\section{LICENCE}

CC BY-NC-ND 4.0

\section{REPOSITORY RECORD}

Hamer, Mark, G. David Batty, and Mika Kivimaki. 2019. "Depressive Symptoms and Obesity: Instrumental Variable Analysis Using Mother-offspring Pairs in the 1970 British Cohort Study". figshare. https://hdl.handle.net/2134/22182. 
1 Depressive symptoms and obesity: Instrumental variable analysis using mother-offspring

2 pairs in the 1970 British Cohort Study

3

4 Mark Hamer, $\mathrm{PhD}^{1,2}$; G. David Batty, $\mathrm{PhD}^{2}$; Mika Kivimaki, $\mathrm{PhD}^{2}$

$5 \quad{ }^{1}$ School of Sport, Exercise \& Health Sciences, Loughborough University; ${ }^{2}$ Department of

6 Epidemiology and Public Health, University College London, London, UK

7

8

9

10

11

12

13

14

15

16

17

18

19

20

21

22

23

24

25

26

27

28

Correspondence to: Professor Mark Hamer; School of Sport, Exercise \& Health Sciences, Loughborough University, Epinal Way, LE11 3TU, United Kingdom

E-mail: m.hamer@lboro.ac.uk; phone: +44-1509-228473 
30 Background: The extent to which depression and obesity are causally related remains to be determined. We utilised intergenerational data on mother - offspring pairs in an instrumental variable analysis to examine the longitudinal association between adolescent depressive symptoms and body mass index (BMI) in adulthood.

Methods: A total of 4,733 mother-offspring pairs were identified from the 1970 British Cohort study. Mothers completed the Malaise Inventory to assess depressive symptoms on three occasions across their offsprings childhood/adolescence (ages 5, 10, 16). Height and weight were recorded in mother and offspring (age 16). Measures of height, weight and the Malaise inventory were repeated in the participant at age 42 .

Results: Maternal malaise score was associated with offspring malaise score, thus confirming the validity of the chosen instrumental variable. A higher mother's malaise score was associated with higher offspring $\mathrm{BMI}$ at follow up $(\mathrm{B}=0.043,95 \% \mathrm{Cl}, 0.013,0.072)$. There was a higher risk of adulthood offspring obesity in mothers with two or three episodes of depression compared with one or none (Odds ratio, 1.42, 95\% Cl, 1.14, 1.76). The maternal malaise - offspring BMI association remained $(p=0.003)$ after adjustment for offspring malaise score, suggesting that maternal mental health influences offspring obesity through mechanisms other than depression. Results from standard and instrumental variable analyses did not support a causal pathway in a direction from BMI to depression.

Conclusions: Our data support a causal pathway linking adolescent depressive symptoms to adiposity in adulthood over 26 years follow up. The reverse direction, ie, adiposity to depression, was not supported.

Key words: Birth cohort; Epidemiology; Obesity; Depression 
53 Obesity and depression are both risk factors for morbidity and mortality, ${ }^{1,2}$ but the extent to which 54 these two conditions are causally related remains to be determined. In particular, understanding the direction of association is one of the problems with the contemporary literature on depression and obesity. There is evidence to suggest excess adiposity might lead to depressive symptoms, ${ }^{3}$ although depressive symptoms may also precede the development of obesity. ${ }^{4-6}$ Since co-occurrence of obesity and depressive symptoms is also possible, this raises difficulties in establishing direction of association in observational studies. Investigators have taken various approaches in an attempt to resolve the direction of association. In studies with data collected across the life course, pre-morbid childhood obesity has been related to the subsequent occurrence of mental health problems. ${ }^{7}$ Such an approach is, however, still subject to residual confounding. Using another technique, investigators have used Mendelian randomization, a form of instrumental variable analysis, to examine the association of obesity with mental health problems. This approach utilises adiposity-related genetic variants as an unconfounded instrument variable for obesity. With fat mass and obesity-associated (FTO) genotype representing a proxy or instrument for higher body mass index (BMI), the rationale is that as different FTO genotypes are randomly allocated at conception, the associations of FTO variants with depression should be free of confounding and reverse causation. This approach has, however, produced inconsistent findings with direct, ${ }^{8,9}$ inverse ${ }^{10}$ and null associations ${ }^{11}$ reported between obesity and later risk of depressive symptoms. With robust genetic variants for depression presently unknown, it has not yet been possible to use Mendelian randomization approaches for assessing the role of depression in the aetiology of obesity.

An alternative instrumental variable analysis may be used in this context. It is well established that maternal (and paternal) BMI is related to offspring $\mathrm{BMI}^{12}$ and the same is known for the intergenerational correlation for mental health. ${ }^{13}$ It is also the case that maternal-offspring 
relationships are likely to be less biased and confounded than conventional analyses using exposure and outcome data from the same individual. For example, illnesses that lead to lower BMI could generate a spurious inverse association between $\mathrm{BMI}$ and depression but it is unlikely to bias the association between mother's BMI and offspring depression. Accordingly, to test the hypothesis that depression increases the risk of obesity, we used a novel instrumental variable analysis (the first of its kind in this area); maternal mental health was used as an instrument for offspring mental health to examine whether it predicted offspring's BMI in adulthood. Since both symptom severity and chronicity of exposure may be important in predicting outcomes, we modelled maternal depression using both absolute symptom scores and also as long term exposure from data on maternal depressive symptoms across three time points. In order to test the hypothesis of obesity leading to depression, we studied the associations of maternal BMI with offspring BMI and subsequent mental health.

\section{Methods}

\section{Design and participants}

The 1970 British Cohort Study (BCS70) follows the lives of 17,284 people born in England, Scotland and Wales in a single week of $1970 .{ }^{14}$ Since birth, participants have been followed up on multiple occasions across their life. The present analyses incorporated data from the age 16 and age 42 year surveys. The age 16 year survey (1986) contained a participant self-completion section on healthrelated behaviours in addition to questions completed by the mother of the participant. The age 42 survey (2012/13) comprised a 60 minute face-to-face computer-assisted-personal-interview, which included a vocabulary task and a self-completion section. At the age 16 survey 6898 participants completed the self-completion module although 9,842 took part in the age 42 survey. The lower response at age 16 arose because of a teachers' strike that resulted in many participants not 
receiving the questionnaires. Participants provided informed consent and all data collection on BCS70 received full ethical approval (London Central REC). Inventory ${ }^{15}$ consisting of items on depressive mood, which has demonstrated acceptable psychometric properties. ${ }^{16}$ The inventory has been validated against external criteria covering current or recent psychiatric morbidity and health service use. ${ }^{17} \mathrm{~A}$ total symptom score, (ranging from 0 to 24 for the 24 item and $0-9$ for the 9 item inventory), was derived from a count of the number of items eliciting a positive response, with higher scores indicating greater severity. At age 16 both the participant and mother completed the Malaise questions and at age 42 the questions were repeated in the participant. At age 16 height and weight were objectively collected in the participant but self-reported in the mother, and at age 42 self reported height and weight were collected in the participant. BMI was calculated using the standard formula (weight $[\mathrm{kg}]$ / height $\left.114\left[\mathrm{~m}^{2}\right]\right)$.

At age 16 respondents were asked questions on smoking (never; rarely; $\geq 1$ cigarette/week), frequency of alcohol intake (daily; 4 -5 /week; 2-3/week; once a week; once a month; rarely; never), time spent in three types of screen based, sedentary activities (TV, video films, computer games) 'after school yesterday' (not at all; less than $1 \mathrm{hr} ;>1 \mathrm{hr} ;>2 \mathrm{hr}$; $>3 \mathrm{hr} ;>4 \mathrm{hr} ;>5 \mathrm{hr}$ ), and serious childhood illness/ accidents/ operations. Parents also provided information on their occupation, which were categorised using the 1970 and 1980 Office of Population Censuses and Surveys

122 Classification of Occupations (managerial/ professional/ intermediate/ routine and manual). 
We examined the distribution of offspring lifestyle and health variables according to tertiles of maternal Malaise score. We estimated coefficients for their association with maternal malaise score using logistic and linear regression. We performed an instrumental-variables regression analysis using two-stage least-squares regression "2SLS" command in SPSS to examine whether maternal Malaise score was associated with offspring BMI through its relationship with offspring Malaise. We compared results from the instrumental-variable estimates of the association between malaise measures and BMI with results from standard linear regression. In order to examine if the so-called exclusion restriction assumption may be violated we further adjusted the model for offspring malaise score (age 16). In addition, we used maternal BMI as an instrumental variable to explore the causal association between offspring's BMI and malaise score in adulthood. Standard linear regression models were adjusted for sex and mothers social status. In order to capture long term exposure, we incorporated measures of maternal malaise from two earlier survey points (age 5 and 10). A depressive case was defined as $>80^{\text {th }}$ centile distribution from malaise scores at each time point (ages 5, 10,16), which was used to generate a grouped exposure variable (never depressed; one occasion; two or three occasions). Logistic regression was used to examine the association between episodes of maternal depression and risk of offspring obesity in adulthood. All analyses were conducted using SPSS version 22.

\section{Results}

Depression and $B M I$ in adulthood

144 A total of 4733 mother-offspring pairs were identified. There were small differences between mothers of offspring included and excluded from these analyses. For example, mothers excluded were more likely to come from lower social occupational classes ( $39.8 \%$ vs. $36.1 \%, p=0.001)$ compared to the analytic sample, although there were no differences in age of mother $(p=0.56)$. On 
average mothers were aged $26.2 \pm 5.4$ yrs at the age 5 survey although mothers' age was not associated with Malaise scores or chronic depressive episodes. At follow-up 42.3\%, 36.2\%, 21.5\% of offspring were categorised as normal weight, overweight, and obese, respectively. Table 1 shows that maternal malaise score was associated with offspring malaise score, thus confirming the validity of the chosen instrumental variable $(F-v a l u e=18.68, p<0.001)$. Maternal malaise was also associated with several potential confounders including offspring smoking and sedentary behaviour. Nevertheless, stronger associations were observed between offspring malaise score and these confounders, in particular for smoking; participants with Malaise scores in the upper tertile compared with lower tertile had $1.8(95 \% \mathrm{Cl}, 1.5,2.1)$ higher odds of smoking, whereas the respective association using mother's Malaise score was weaker ( $\mathrm{OR}=1.4,95 \% \mathrm{Cl}, 1.2,1.7)$.

We found a linear association between episodes of depression and mothers' own BMI in models adjusted for social status and age, such that higher $B M I$ was observed in mothers with one $(B=0.33$, $\left.95 \% \mathrm{Cl}, 0.08,0.58 \mathrm{Kg} / \mathrm{m}^{2}\right)$ and two or more episodes $\left(\mathrm{B}=0.68,95 \% \mathrm{Cl}, 0.40,0.96 \mathrm{Kg} / \mathrm{m}^{2}\right)$ compared to mothers never depressed. In longitudinal analysis examining associations between malaise and offspring $\mathrm{BMI}$ at follow up, associations using offspring malaise age 16 were attenuated to the null after adjustment for mother's social class. A higher mother's malaise score, however, remained associated with higher offspring BMI at follow up (Table 2). More robust effect estimates were observed in the instrumental variable analysis. In analyses to explore the so-called exclusion restriction assumption adjustment for offspring malaise score age 16 did not alter the maternal malaise - offspring $\mathrm{BMI}$ association $(\mathrm{B}=0.063,95 \% \mathrm{Cl}, 0.021,0.105, \mathrm{p}=0.003)$. In view of the findings showing associations between our instrumental variable and possible confounders we also conducted a further analysis that controlled for offspring smoking and sedentary behaviours (at age 16) although this did not influence the association between maternal depression and offspring BMI

171 $(B=0.065,95 \% \mathrm{Cl}, 0.015,0.115, p=0.01)$. 
In analyses to examine the association between episodes of maternal depression and risk of offspring obesity in adulthood we observed a dose-response association, with a higher risk of offspring obesity in mothers with two or three episodes of depression compared with one or none 175 (Table 3).

BMI and depression in adulthood

177 We also used maternal BMI as an instrumental variable to explore the causal association between offspring's BMI and malaise score in adulthood. Maternal BMI was strongly associated with offspring $\mathrm{BMI}(\mathrm{F}$-value $=58.7, \mathrm{p}<0.001)$, supporting the use of maternal $\mathrm{BMI}$ as a valid instrument for offspring BMI. However, maternal BMI was also associated with several potential confounders including offspring smoking (OR per unit increase $=1.03,95 \% \mathrm{Cl}, 1.01-1.06, \mathrm{p}=0.015)$ and sedentary behaviour $(\mathrm{OR}=1.05,95 \% \mathrm{Cl}, 1.02-1.08, \mathrm{p}=0.001)$. Results from standard and instrumental variable analyses did not support a causal pathway in a direction from BMI to depression (Table 4). In further analysis that controlled for offspring smoking and sedentary behaviours (at age 16) the association between $\mathrm{BMI}$ and depression was unchanged $(\mathrm{B}=0.013,95 \% \mathrm{Cl},-0.008,0.035, \mathrm{p}=0.23)$.

\section{Discussion}

The aim of this study was to examine associations between depressive symptoms and obesity across the life course using an instrumental variable approach. Existing instrumental variable analyses in this field have used the FTO and other genotypes as an unconfounded measure of obesity. However, as robust genetic variants for depression are presently unknown, we chose to adopt a novel approach utilising intergenerational data on mother - offspring pairs for assessing the role of depression in the aetiology of obesity. We found evidence to support a causal pathway linking adolescent depressive symptoms to BMI in adulthood over 26 years follow up. The reverse direction, ie, BMI to depression, was not supported. 
Our instrumental variable, maternal depressive symptoms, met the majority of assumptions required for an instrumental variables analysis. Firstly, in our study and other published studies, ${ }^{13}$ maternal depressive symptoms were positively associated with offspring depressive symptoms. Second, an offspring's body mass index in adulthood (outcome) cannot plausibly affect variation in maternal depressive symptoms. Thus, our instrumental variable analysis was more protected from reverse causality than conventional analysis. The final assumption postulates that except from its association with the risk factor of interest, there is no other pathway linking the instrumental variable with the outcome of interest. This assumption was only partly met, as both maternal mental health and BMI was associated with several factors (offspring sedentary behaviour and smoking status) which might be associated with the respective outcomes in offspring. However, the maternal influence on these confounding factors is likely to be weaker than that of participant's, as confirmed in the present study. Maternal depressive symptoms might contribute to an environment that may likely to be diluted.

After adjustment for offspring malaise score, the maternal malaise-BMI association persisted, thus providing further evidence for violation of the exclusion restriction assumption and suggesting that maternal mental health may influence offspring obesity through mechanisms other than depression. For example, genetic vulnerability to depression may be associated with pleiotropic effects reflecting unknown biological pathways driving BMI of offspring in later life. In addition, children with depressed mothers may be exposed to more environmental and family risk factors, than families without depression, ${ }^{13}$ although we attempted to control for this by adjusting for mother's social status. 
221 A direct pathway from depression to obesity seems biologically plausible. One of the driving factors

222

223

224

225

226

227

231

might be disturbances in key stress axes including the hypothalamic pituitary adrenal axis and sympathetic nervous system. Disturbances in these axes have been associated with depressive symptoms ${ }^{18}$ and are linked to insulin resistance and the cascade of events in the metabolic syndrome, including deposition of adiposity. ${ }^{19-21}$ Furthermore, depression is associated with reduced physical activity, ${ }^{22}$ which may also contribute to increased weight gain. These direct and indirect underlying mechanisms may conceivably operate over a prolonged period, thus studies with sufficient follow up would be required to detect the effects and may therefore explain the equivocal nature of the literature.

The main strength of this study is the use of intergenerational, longitudinal data to reduce confounding and bias. In addition we utilised data on maternal depressive symptoms across three time points during their offspring's childhood/adolescence in order to better capture long term exposure. The limitations included the use of self-reported weight and height. Previous studies, however, have demonstrated the validity of using self-reported weight. ${ }^{23}$ Furthermore, errors in selfreported weight are often systematic instead of random, reflecting both rounding to the nearest point of heaping and a tendency to report weights closer to ideal weight. ${ }^{24}$ Although well validated, the instrument used to assess depressive symptoms could not capture clinical diagnosis. The Malaise Inventory is essentially a measure of 'negative affect' since it covers an array of items relating to anxiety disorder, psychological and somatic symptoms. Although the Malaise inventory has not been commonly used in the past, a wide variety of psychometric instruments have been employed to assess negative affect (commonly termed as 'depressive symptoms') in this area. Depressive symptoms were measured using different versions (24-item and 9-item) of the Malaise Inventory at various stages of the survey although the full 24 item version is known to be more reliable. In addition, different subtypes of depression may be more strongly associated with obesity. ${ }^{25}$ Missing 
data may have introduced bias as excluded participants were more disadvantaged. Nevertheless, given that this may have slightly constrained variation in depressive symptoms and BMI our results

248 are likely to reflect conservative estimates. Over 95\% of the cohort was 'British white' thus it was

249 not possible to investigate possible interactions by ethnicity. Although our study does not provide a

250 definitive answer to the complexities in the relationships between depression and obesity, it adds a

251 novel component to the cumulating evidence on depression as a long-term risk factor for increased

252 body mass.

253

254 In summary, to our knowledge this is the first study to adopt a novel approach utilising

255 intergenerational data on mother - offspring pairs to examine a causal pathway linking adolescent

256 depressive symptoms to BMI in adulthood.

257

258

259 


\section{Funding}

The data were made available through the UK Data Archive. MK is supported by the MRC (K013351), ESRC and NordForsk. MH acknowledges support from the NIHR Leicester-Loughborough Diet, Lifestyle and Physical Activity Biomedical Research Unit, which is a partnership between University Hospitals of Leicester NHS Trust, Loughborough University and the University of Leicester. The funders had no role in the study design; in the collection, analysis and interpretation of data; in writing of the report; or in the decision to submit the paper for publication.

\section{Author contributions}

$\mathrm{MH}$ had full access to the data, and takes responsibility for the integrity and accuracy of the results. All authors contributed to the concept and design of study, drafting and critical revision of the manuscript.

\section{Conflict of interest}

None of the authors have any competing interests to declare. 


\section{References}

1. Flegal KM, Kit BK, Orpana H, Graubard BI. Association of all-cause mortality with overweight and obesity using standard body mass index categories: a systematic review and meta-analysis. JAMA. 2013;309(1):71-82.

2. Gallo JJ, Morales KH, Bogner HR, Raue PJ, Zee J, Bruce ML, et al. Long term effect of depression care management on mortality in older adults: follow-up of cluster randomized clinical trial in primary care. BMJ. 2013;346:f2570.

3. Luppino FS, de Wit LM, Bouvy PF, Stijnen T, Cuijpers P, Penninx BW et al. Overweight, obesity, and depression: a systematic review and meta-analysis of longitudinal studies. Arch Gen Psychiatry. 2010;67:220-9.

4. Liem ET, Sauer PJ, Oldehinkel AJ, Stolk RP. Association between depressive symptoms in childhood and adolescence and overweight in later life: review of the recent literature. Arch Pediatr Adolesc Med. 2008 Oct;162(10):981-8.

5. Kivimäki M, Lawlor DA, Singh-Manoux A, Batty GD, Ferrie JE, Shipley MJ, et al. Common mental disorder and obesity: insight from four repeat measures over 19 years: prospective Whitehall II cohort study. BMJ. 2009;339: b3765.

6. Fezeu LK, Batty GD, Gale CR, Kivimaki M, Hercberg S, Czernichow S. Is the Relationship between Common Mental Disorder and Adiposity Bidirectional? Prospective Analyses of a UK General Population-Based Study. PLoS One. 2015;10(5):e0119970.

7. Viner RM, Cole TJ. Adult socioeconomic, educational, social, and psychological outcomes of childhood obesity: a national birth cohort study. BMJ. 2005;330(7504):1354.

8. Kivimäki M, Jokela M, Hamer M, Geddes J, Ebmeier K, Kumari M et al. Examining overweight and obesity as risk factors for common mental disorders using fat mass and obesity-associated (FTO) genotype-instrumented analysis: The Whitehall II Study, 19852004. Am J Epidemiol. 2011;173:421-9.

9. Rivera M, Cohen-Woods S, Kapur K, Breen G, Ng MY, Butler AW, et al. Depressive disorder moderates the effect of the FTO gene on body mass index. Mol Psychiatry. $2012 ; 17(6): 604-11$.

10. Lawlor DA, Harbord R, Tybaerg-Hansen A. Using genetic loci to understand the relationship between adiposity and psychological distress: a Mendelian Randomization study in the Copenhagen General Population study of 53,221 adults. J Intern Med 2011; 269: 525-37.

11. Walter S, Kubzansky LD, Koenen KC, Liang L, Tchetgen EJ, Cornelis MC, et al. Revisiting Mendelian randomization studies of the effect of body mass index on depression. Am J Med Genet B Neuropsychiatr Genet. 2015;168B(2):108-15.

12. Perez-Pastor EM, Metcalf BS, Hosking J, Jeffery AN, Voss LD, Wilkin TJ. Assortative weight gain in mother-daughter and father-son pairs: an emerging source of childhood obesity. Longitudinal study of trios (EarlyBird 43). Int J Obes (Lond). 2009;33(7):727-35.

13. Barker ED, Copeland W, Maughan B, Jaffee SR, Uher R. Relative impact of maternal depression and associated risk factors on offspring psychopathology. Br J Psych 2012;200:124-129.

14. Elliott J, Shepherd P. Cohort profile: 1970 British Birth Cohort (BCS70). Int J Epidemiol 2006;35:836-43. 
15. Rutter M, Tizard J, Whitmore K. Education, health and behaviour. London: Longmans, 1970.

16. McGee R, Williams S, Silva PA. An evaluation of the Malaise Inventory. J Psychosom Res. 1986;30(2):147-52.

17. Rodgers B, Pickles A, Power C, Collishaw S, Maughan B. Validity of the Malaise Inventory in general population samples. Soc Psychiatry Psychiatr Epidemiol. 1999;34(6):333-41.

18. Koenigsberg HW, Teicher MH, Mitropoulou V, Navalta C, New AS, Trestman R et al. 24-h Monitoring of plasma norepinephrine, MHPG, cortisol, growth hormone and prolactin in depression. J Psych Res 2004;38:503-11.

19. Licht CM, Vreeburg SA, van Reedt Dortland AK. Increased sympathetic and decreased parasympathetic activity rather than changes in hypothalamic-pituitary-adrenal axis activity is associated with metabolic abnormalities. J Clin Endocrinol Metab. 2010;95: 2458-66.

20. Mancia G, Bousquet P, Elghozi JL, Esler M, Grassi G, Julius S. The sympathetic nervous system and the metabolic syndrome. J Hypertens. 2007;25: 909-920.

21. Rosmond R, Dallman MF, Björntorp P. Stress-related cortisol secretion in men: relationships with abdominal obesity and endocrine, metabolic and hemodynamic abnormalities. J Clin Endocrinol Metab. 1998;83:1853-9.

22. Azevedo Da Silva M, Singh-Manoux A, Brunner EJ, Kaffashian S, Shipley MJ, Kivimäki M, et al. Bidirectional association between physical activity and symptoms of anxiety and depression: the Whitehall II study. Eur J Epidemiol. 2012;27(7):537-46.

23. Lowry R, Galuska DA, Fulton JE, Wechsler H, Kann L, Collins JL. Physical activity, food choice, and weight management goals and practices among US college students. Am J Prev Med. 2000;18(1):18-27.

24. Rowland ML. Self-reported weight and height. Am J Clin Nutr. 1990;52(6):1125-33.

25. Lasserre AM, Glaus J, Vandeleur CL, Marques-Vidal P, Vaucher J, Bastardot F, et al. Depression with atypical features and increase in obesity, body mass index, waist circumference, and fat mass: a prospective, population-based study. JAMA Psychiatry. 2014; $71(8): 880-8$. 